\title{
Impact of LHC data on muon $g-2$ solutions in vector-like extension of the Constrained MSSM
}

\author{
Arghya Choudhury, ${ }^{a}$ Soumya Rao, ${ }^{b}$ and Leszek Roszkowski ${ }^{a, b}$ \\ a Consortium for Fundamental Physics, Department of Physics and Astronomy, \\ University of Sheffield, Sheffield S3 $7 R H$, United Kingdom \\ ${ }^{b}$ National Centre for Nuclear Research, \\ Hoża 69, 00-681 Warsaw, Poland \\ E-mail: a.choudhury@sheffield.ac.uk, soumya.rao@ncbj.gov.pl, \\ leszek.roszkowski@ncbj.gov.pl
}

ABSTRACT: The long-standing discrepancy between the experimental determination by the Muon $g-2$ Collaboration at Brookhaven and the Standard Model predictions for the anomalous magnetic moment of the muon cannot be explained within simple unified framework like the Constrained Minimal Supersymmetric Standard Model, but it can within its extension with vector-like fermions. In this paper we consider a model with an additional vector-like $5+\overline{5}$ pair of $S U(5)$. Within this model we first identify its parameter space that is consistent with the current discrepancy and show that this implies the lighter chargino mass in the range of $700-1200 \mathrm{GeV}$. We examine how it is affected by constraints from electroweak sparticle search at the LHC based on $13 \mathrm{TeV}$ search with $36.1 \mathrm{fb}^{-1}$ integrated luminosity. We show that null trilepton signal searches coming from chargino-neutralino pair production significantly constrains the allowed parameter space except when the charginoneutralino mass difference is relatively small, below about $10 \mathrm{GeV}$. Next we consider the expected impact of the New Muon $g-2$ experiment at Fermilab with its projected sensitivity reach of $7 \sigma$ and, assuming it confirms the current discrepancy, show that the remaining parameter space of the considered model will be in strong tension with the current LHC limits. 


\section{Contents}

1 Introduction 1

2 Vector like extension of the CMSSM 3

3 Constraints from flavor physics, $(g-2)_{\mu}$ and direct detection of DM 4

4 Allowed parameter space and decay properties of EWinos 5

4.1 Decay modes for $\chi_{2}^{0}$ : 6

4.2 Decay modes for $\chi_{1}^{ \pm}$: $\quad 7$

4.3 Benchmark points and models 8

5 Collider analysis for trilepton searches $\quad 8$

$\begin{array}{ll}5.1 & \text { Validation for trilepton analysis } \\ 5.2 & \text { New }\end{array}$

$\begin{array}{ll}5.2 \text { New limits for benchmark models } & 12\end{array}$

6 Conclusions 13

\section{Introduction}

Despite the absence of a signal for supersymmetry (SUSY) at the LHC, it still remains one of the most appealing frameworks for physics beyond the Standard Model (BSM). Besides providing a natural candidate for dark matter (DM), it also gives possible explanation for the discrepancy that exists in the Standard Model (SM) value of the anomalous magnetic moment of muon, $(g-2)_{\mu}$, and the experimentally measured quantity. The SM value for $(g-2)_{\mu}$ differs by more than $3 \sigma$ from the measured value $[1,2]$. Future measurement at Fermilab $[3,4]$ is expected to improve the sensitivity of the previous measurement by a factor of four and hence potentially confirm or falsify the persistent disagreement. In SUSY, the explanation for the difference arises from contributions due to smuon-neutralino and sneutrino-chargino loops. To fit the $(g-2)_{\mu}$ anomaly within the framework of the minimal supersymmetric Standard Model (MSSM), one requires the slepton and the lighter chargino masses in a range of a few hundreds of $\mathrm{GeV}[5-11]$. However, the stringent bounds on the strong sector (squarks and gluinos) from the LHC and the Higgs mass measurements rule out the possibility of explaining $(g-2)_{\mu}$ in GUT-constrained models like the Constrained MSSM (CMSSM) and the Non-Universal Higgs Mass (NUHM) model [12-14]. The way out has usually been to assume non-universal gaugino masses [15-22] which can provide a viable parameter space to explain $(g-2)_{\mu}$ while at the same time not conflicting with constraints from LEP and LHC.

There have also been alternative solutions as for example, adding vector-like (VL) matter to MSSM which has been studied in Refs. [23-32]. The presence of new VL sector 
gives extra contribution to $(g-2)_{\mu}$ by introducing new sources of smuon mixing and new Yukawa couplings [32]. Apart from $(g-2)_{\mu}$, it has been shown that VL colored sparticles provide extra contributions to Higgs mass [33-38] and several phenomenological analyses have addressed the extra VL matter in the context of various long-standing theoretical issues related to beyond SM physics [23-32].

In particular, Ref. [32] studied two simple extensions of the CMSSM by adding a pair of multiplets, firstly in the $\mathbf{5}+\overline{\mathbf{5}}$ and secondly in the $\mathbf{1 0}+\overline{\mathbf{1 0}}$ representations of $S U(5)$. It was shown that the model could satisfy various constraints from flavor physics and LHC direct searches, as well as include a viable dark matter candidate that was in agreement with relic density and direct detection constraints, for a considerable region of the parameter space [32]. In particular, through the additional mixing of VL fields with second generation leptons, the model proved particularly useful in explaining the $(g-2)_{\mu}$ discrepancy. In this work we extend the analysis considered in Ref. [32], using the models with additional $\mathbf{5}+\overline{\mathbf{5}}$. Motivated by the solution to the $(g-2)_{\mu}$ discrepancy, we examine the impact of collider constraints on the viable parameter space.

As mentioned earlier, the allowed parameter spaces satisfying $(g-2)_{\mu}$ constraints are characterised by light electroweak (EW) sparticles, i.e., light EW gauginos or electroweakinos (the charginos and the neutralinos) and charged sleptons. Hence to probe the relevant parameter space at the LHC, the most sensitive search is chargino and neutralino pair production (via $p p \rightarrow \chi_{1}^{ \pm} \chi_{2}^{0}$ ) leading to the trilepton + transverse missing energy $\left(\mathrm{E}_{\mathrm{T}}\right.$ ) signal. Both CMS and ATLAS Collaborations have looked for electroweakinos, or EWinos, with different leptonic final states [39-47], among which the trilepton data gives the most stringent bound. From the very recent LHC analysis of Run-II data with $\mathcal{L}=36.1 \mathrm{fb}^{-1}$, ATLAS has excluded chargino masses upto $1150 \mathrm{GeV}$ for relatively light LSP [46]. However, ATLAS and CMS have presented these limits for a few particular type of simplified models with specific assumptions on the compositions and branching ratios of EWinos. The electroweakinos searches and related topics in the context of the LHC have been analysed by various phenomenology group in Ref. [7, 9, 48-66]. Due to the presence of VL particles and their mixing with SM, the electroweakinos (mainly $\chi_{1}^{ \pm}, \chi_{2}^{0}$ ) can have non standard branching ratios compared to the CMSSM or usual phenomenological MSSM (pMSSM) scenarios. Hence the limits interpreted by ATLAS or CMS for various simplified models are not directly applicable to such models and a reinterpretation of the bounds from trilepton searches at the LHC is necessary.

The paper is organized as follows. We first give a brief overview of the model which is obtained by adding a VL $\mathbf{5}+\overline{\mathbf{5}}$ of $S U(5)$ pair to CMSSM in Section 2. We briefly mention the constraints applied to obtain the relevant allowed parameter space in Section 3 and then discuss different scenarios for chargino $\left(\chi_{1}^{ \pm}\right)$and neutralino $\left(\chi_{2}^{0}\right)$ decays in the context of VL extension of CMSSM in Section 4. In Section 5, we show results for LHC trilepton searches from chargino-neutralino pair production using the latest LHC Run-II $36.1 \mathrm{fb}^{-1}$ data. Finally, we give our conclusions in Section 6. 


\section{Vector like extension of the CMSSM}

We follow the model studied and analysed in [32], particularly in the context of $(g-2)_{\mu}$ where the MSSM is extended through the addition of a pair $\mathbf{5}+\overline{\mathbf{5}}$ or a pair $\mathbf{1 0}+\overline{\mathbf{1 0}}$. However, it was shown in [32] that the $\mathbf{1 0}+\overline{\mathbf{1 0}}$ extension was more fine tuned in order to provide a viable parameter space for a significant contribution to $(g-2)_{\mu}$ and therefore the analysis was restricted to $\mathbf{5}+\overline{\mathbf{5}}$. Here we shall focus only on the $\mathbf{5}+\overline{\mathbf{5}}$ extension, which we shall from now on refer to as the LD model following the previous convention. We summarize the main features of the LD model below (for more details see Ref. [32]).

The LD model consists of extending the MSSM spectrum with the addition of the following fields: ${ }^{1}$

$$
\begin{aligned}
D & =(\overline{\mathbf{3}}, \mathbf{1}, 1 / 3) & D^{\prime} & =(\mathbf{3}, \mathbf{1},-1 / 3) \\
L & =(\mathbf{1}, \mathbf{2},-1 / 2) & L^{\prime} & =(\mathbf{1}, \mathbf{2}, 1 / 2) .
\end{aligned}
$$

This implies the addition of a quark with charge $-1 / 3$ and a charged lepton along with their antiparticles, and two massive neutrinos to the MSSM spectrum. Correspondingly the sparticle content sees the addition of squarks, sleptons and sneutrinos.

In comparison to the MSSM, there are now additional trilinear and bilinear terms in the superpotential,

$$
W \supset-\lambda_{D} q H_{d} D-\lambda_{L} L H_{d} e+M_{D} D D^{\prime}+M_{L} L L^{\prime}+\widetilde{M}_{L} l L^{\prime}+\widetilde{M}_{D} d D^{\prime},
$$

Finally the soft SUSY-breaking Lagrangian also has extra terms involving $\tilde{L}^{(\prime)}$ and $\tilde{D}^{(\prime)}$ as follows

$$
\begin{aligned}
-\mathcal{L}_{\text {soft }} \supset & {\left[m_{L}^{2}|\tilde{L}|^{2}+m_{L^{\prime}}^{2}\left|\tilde{L}^{\prime}\right|^{2}+m_{D}^{2}|\tilde{D}|^{2}+m_{D^{\prime}}^{2}\left|\tilde{D}^{\prime}\right|^{2}+\left(\widetilde{m}_{L}^{2} \tilde{l}^{\dagger} \tilde{L}+\widetilde{m}_{D}^{2} \tilde{d}^{\dagger} \tilde{D}+\text { h.c. }\right)\right] } \\
& +\left(B_{M_{L}} \tilde{L} \tilde{L}^{\prime}+B_{\widetilde{M}_{L}} \tilde{l} \tilde{L}^{\prime}+B_{M_{D}} \tilde{D} \tilde{D}^{\prime}+B_{\widetilde{M}_{D}} \tilde{d} \tilde{D}^{\prime}+\text { h.c. }\right) \\
& -\left(T_{D} \tilde{q} H_{d} \tilde{D}^{\dagger}+T_{L} \tilde{L} H_{d} \tilde{e}^{\dagger}+\text { h.c. }\right)
\end{aligned}
$$

where $\widetilde{m}_{L}^{2}, \widetilde{m}_{D}^{2}, T_{L}, T_{D}, B_{\widetilde{M}_{L}}$, and $B_{\widetilde{M}_{D}}$ are 3-dimensional matrices that govern mixing between MSSM and VL matter. This mixing plays an important role for $(g-2)_{\mu}$ phenomenology.

In addition to the above, we also make the choice of GUT scale parameters such that the boundary conditions for the extra Yukawa couplings demanded by UV completion at the GUT scale are given by

$$
\lambda_{L}=\left(\begin{array}{c}
0 \\
\lambda_{5} \\
\epsilon \lambda_{5}
\end{array}\right),
$$

where $\epsilon<1$. This in turn means that the soft mass matrices which also satisfy the same flavor constraints as the Yukawa couplings, will have their off-diagonal mixing terms parametrized similar to Eqn. 2.3 as follows

\footnotetext{
${ }^{1}$ The MSSM fields are $q=(3,2,1 / 6), u=(\overline{3}, 1,-2 / 3), d=(\overline{3}, 1,1 / 3), l=(\mathbf{1}, \mathbf{2},-1 / 2), e=(\mathbf{1}, \mathbf{2},-1 / 2), H_{u}=(1,2,1 / 2)$, $H_{d}=(1,2,-1 / 2)$ with $S U(3) \times S U(2) \times U(1)$ quantum numbers in parentheses.
} 


$$
\widetilde{m}_{L}^{2}=\widetilde{m}_{D}^{2}=\left(\begin{array}{c}
0 \\
\widetilde{m}^{2} \\
\alpha \widetilde{m}^{2}
\end{array}\right)
$$

where once again $\alpha<1$.

Thus the first generation mixing is almost absent compared to second and third generation mixing. Eqns. 2.3 and 2.4 not only impact the $(g-2)_{\mu}$ contribution but also have a significant effect on the trilepton signal from chargino and neutralino decays as we shall see in Section 4.

\section{Constraints from flavor physics, $(g-2)_{\mu}$ and direct detection of DM}

In this section we mention the GUT scale input parameters used as well as the constraints applied in order to obtain the parameter space shown in Fig. $1^{2}$. The parameter space was scanned using MultiNest [67] and the SARAH [68-71] package was used to generate the spectrum, while the relevant flavor constraints were calculated using the SARAH package FlavorKit [72]. In addition, dark matter constraints on relic density and direct detection were obtained using micrOMEGAs v.3.5.5 [73]. Bounds on the Higgs sector from LHC searches for Higgs production channels, branching ratios as well as Higgs decay were applied using the codes HiggsSignals [74] and HiggsBounds [75-77]. The following ranges of values for the GUT scale input parameters were used, which are also listed in [32]:

VL Yukawa coupling, $\lambda_{5} \in[-0.5,0.5]$,

Yukawa hierarchy factor, $\epsilon \in[-0.5,0.5]$, superpotential mass VL fields, $M_{V} \in[50,1500] \mathrm{GeV}$, superpotential mass mixing, $\widetilde{M} \in[-20,20] \mathrm{GeV}$, mass mixing hierarchy factor, $\alpha \in[0.01,1)$, scalar mass, $m_{0} \in[100,4000] \mathrm{GeV}$, gaugino mass, $m_{1 / 2} \in[300,4000] \mathrm{GeV}$, soft mass mixing, $\widetilde{m}^{2} \in\left[-5 \times 10^{6}, 5 \times 10^{6}\right] \mathrm{GeV}^{2}$, trilinear coupling, $A_{0} \in[-4000,4000] \mathrm{GeV}$, soft bilinear term VL fields, $B_{0} \in[-1500,1500] \mathrm{GeV}$, ratio of the Higgs vevs, $\tan \beta \in[1,60]$,

and the sign of the Higgs mass parameter, sgn $\mu=+1$.

The experimental constraints used to derive the parameter space in addition to the Higgs bounds are flavor physics constraints such as $\mathrm{BR}\left(\bar{B} \rightarrow X_{s} \gamma\right)$ [78], $\mathrm{BR}\left(B_{u} \rightarrow \tau \nu\right)$ [79], $\Delta M_{B_{s}}$ [80], $\Delta \rho$ [80], $\mathrm{BR}\left(B_{s} \rightarrow \mu^{+} \mu^{-}\right)[81,82]$ and $\mathrm{BR}\left(\tau^{ \pm} \rightarrow \mu^{ \pm} \gamma\right)$ [83], while in DM sector

\footnotetext{
${ }^{2}$ The result shown in Fig. 1 is obtained using the same numerical tools and priors used in Ref. [32].
} 
the constraint on relic abundance [84], $\Omega_{\chi} h^{2}$, and the current LUX limit [85] on the spinindependent DM-nucleon scattering cross section, $\sigma_{p}^{\mathrm{SI}}$, are taken into account. For more details on ranges and theoretical and experimental errors see Table 1 of ref. [32].

\section{Allowed parameter space and decay properties of EWinos}

In this section we study decay properties of $\chi_{1}^{ \pm}$and $\chi_{2}^{0}$ in the parameter space which satisfies the constraints mentioned in the previous section as well as $\delta(g-2)_{\mu}$ bounds $(2 \sigma)$ as shown in Fig. 1. The $2 \sigma$ allowed region for $\delta(g-2)_{\mu}$ according to the latest data $[1,2]$ is indicated by the blue solid lines, while the dashed lines indicate future measurement $[3,4]$ with four times greater sensitivity, assuming that the central value remains the same. We consider the red points that are allowed by current $\delta(g-2)_{\mu}$ bounds for further analysis. The trilepton final states from direct chargino-neutralino pair production will be the most effective channel to probe all these points. Due to the choice of input parameters and mixing in our model, it is expected that the decay modes of EWinos could be different from the MSSM cases or usual choices made by ATLAS/CMS with simplified scenarios.

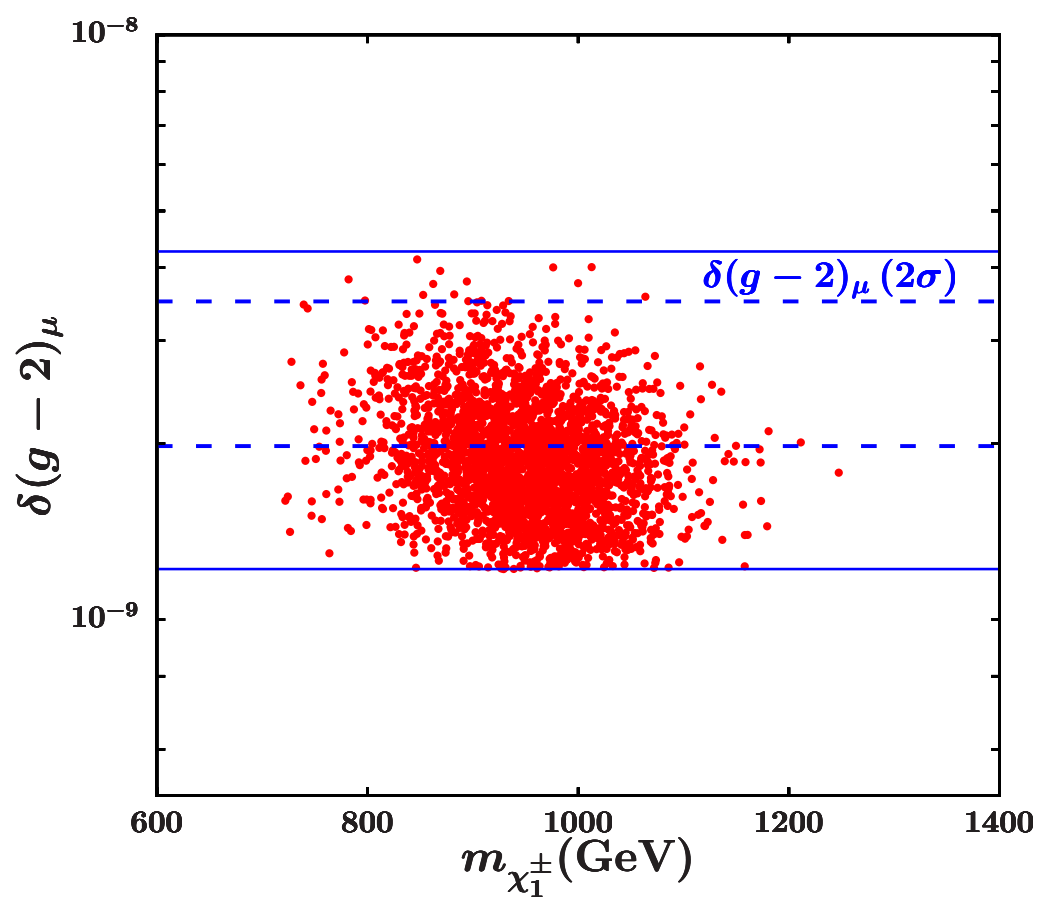

Figure 1: Allowed parameter space for $\delta(g-2)_{\mu}$ in the LD model as a function of chargino mass. The $2 \sigma$ allowed region for $\delta(g-2)_{\mu}$ according to the latest data [1,2] is indicated by the blue solid lines, while the dashed lines indicate future measurement $[3,4]$ with four times greater sensitivity, assuming that the central value remains the same. 

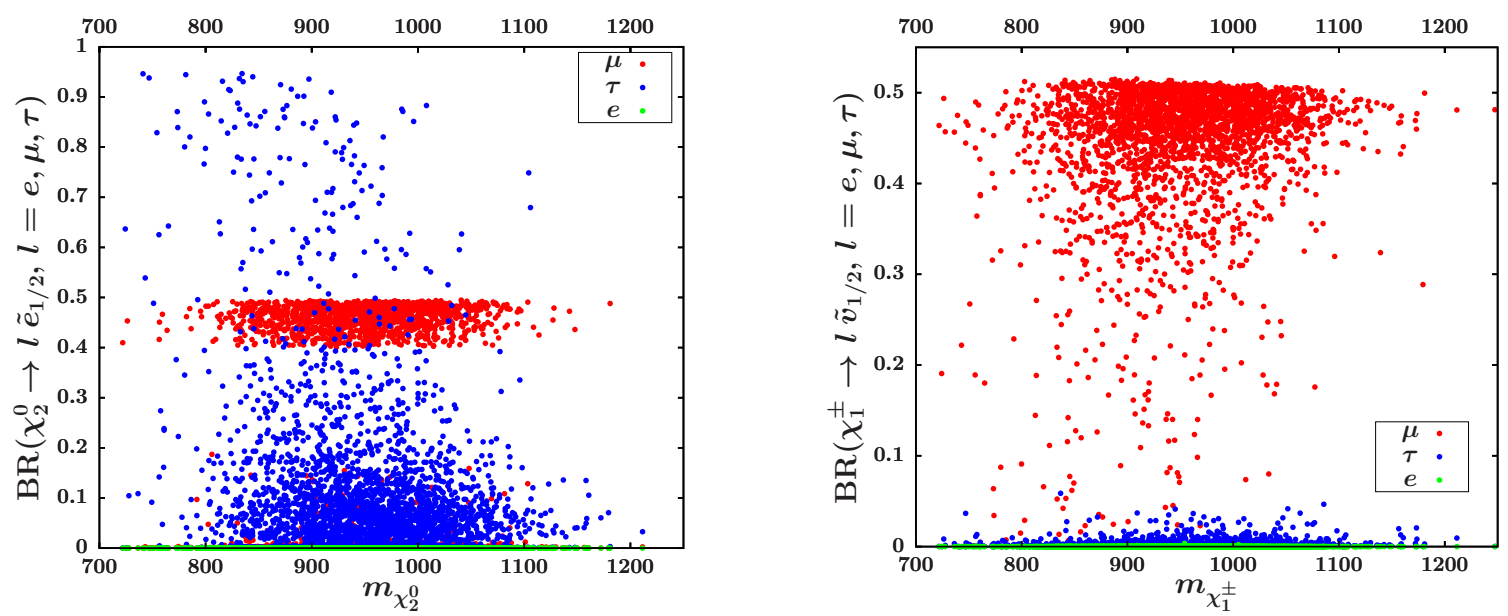

Figure 2: Branching ratio of $\chi_{2}^{0} \rightarrow l \tilde{e}$ (left panel) and $\chi_{1}^{ \pm} \rightarrow l \tilde{\nu}$ (right panel) plotted against $\chi_{2}^{0}$ and $\chi_{1}^{ \pm}$masses, respectively, for the parameter space satisfying the constraints described in the text as well as giving a $(g-2)_{\mu}$ contribution that is within the current $2 \sigma$ limit.

\subsection{Decay modes for $\chi_{2}^{0}$ :}

In general for light slepton scenarios (lighter than $m_{\chi_{1}^{ \pm}}$), the second lightest neutralino $\chi_{2}^{0}$ decays into 2 body final state $-l \tilde{l}$ and $\nu \tilde{\nu}$ where $l$ denotes for $e, \mu$ and $\tau$. For our model, the first slepton mass eigenstate is mostly mixed smuon/VL and the second slepton eigenstate is usually right-handed stau. Hence we sometimes obtain large mass splitting between the first two slepton mass eigenstates. For a significant portion of the parameter space $\chi_{2}^{0}$ decays to a muon and a slepton at $50 \%$ branching ratio, or one sees the $\tau$ lepton channel but no electrons, with $50 \%$ branching ratio for invisible modes. Thus the flavour democratic simplified model scenarios are mostly absent in our model. As a result, apart from the invisible modes which have a $50 \%$ branching ratio, $\chi_{2}^{0}$ can dominantly decay either into $\mu \tilde{\mu}$ or $\tau \tilde{\tau}$ with $50 \%$ branching ratios. For the first case the usual LHC limit will then put more stringent bounds.

In the left panel of Fig. 2, the branching ratios for different leptonic decay modes are plotted against the $\chi_{2}^{0}$ mass. The muon channel is always at $50 \%$ branching ratio but the $\tau$ channel has a branching ratio which is mostly less than $20 \%$ with some points having branching ratio in the range between $20-100 \%$ while no electrons are seen.

In Fig. 3, we present the branching ratios of $\chi_{2}^{0}$ which decays into a muon and a slepton $\left(\tilde{e}_{1} / \tilde{e}_{2}\right)$, where the slepton further decays into a muon and an LSP with $100 \%$ branching ratio. The points are color coded according to the mass differences $m_{\tilde{e}}-m_{\chi_{1}^{0}}$ (left panel) and $m_{\chi_{2}^{0}}-m_{\tilde{e}}$ (right panel). These $\operatorname{BRs}\left(\chi_{2}^{0} \rightarrow \mu \tilde{e}_{1 / 2} \rightarrow \mu \mu \chi_{1}^{0}\right)$ vary within $40-50 \%$ with the rest being invisible, where the slepton could be degenerate with either $\chi_{1}^{0}$ or $\chi_{2}^{0}$. 

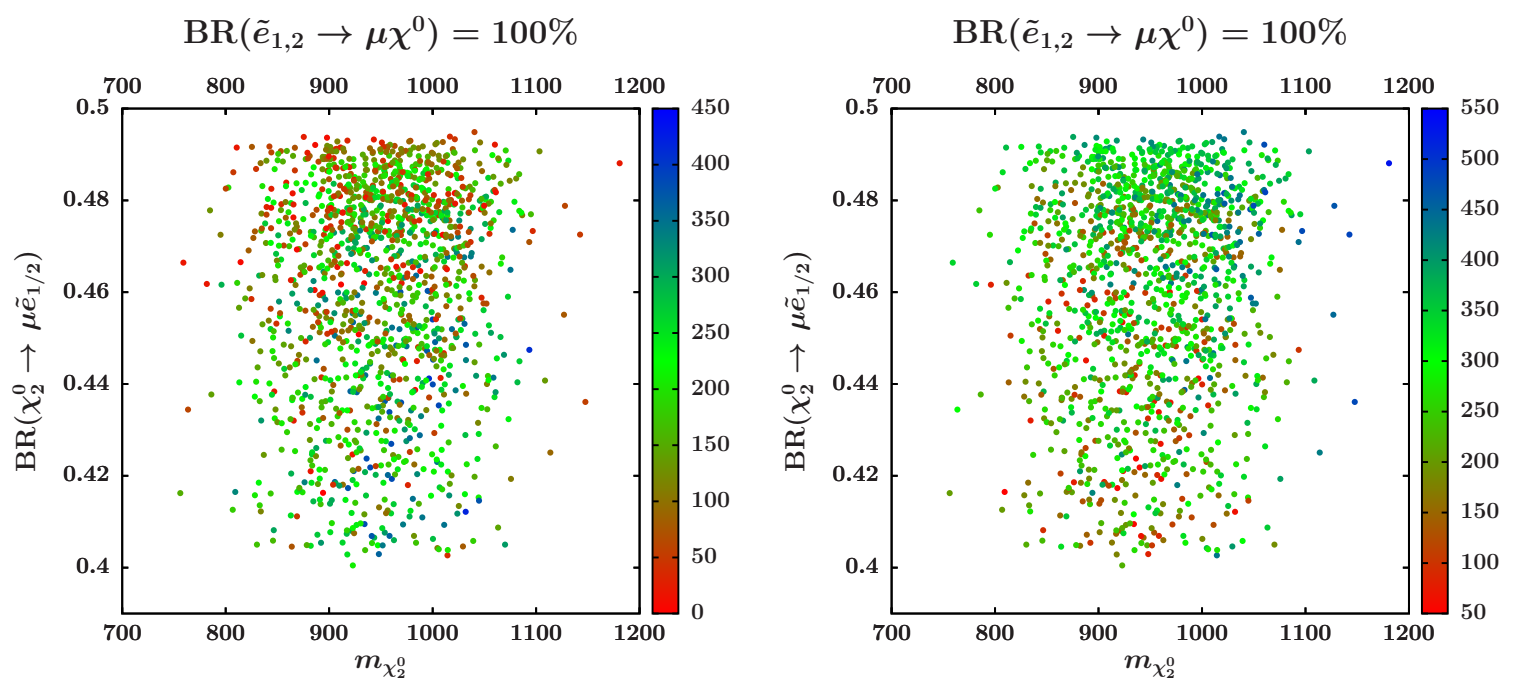

Figure 3: Branching ratio of $\chi_{2}^{0} \rightarrow \mu \tilde{e}$ plotted against $\chi_{2}^{0}$ mass for parameter space satisfying the constraints described in the text as well as giving a $(g-2)_{\mu}$ contribution that is within the current $2 \sigma$ limit. The left panel shows the points color coded with slepton-LSP mass difference while the right panel shows them color coded according to the $\chi_{2}^{0}$-slepton mass difference.
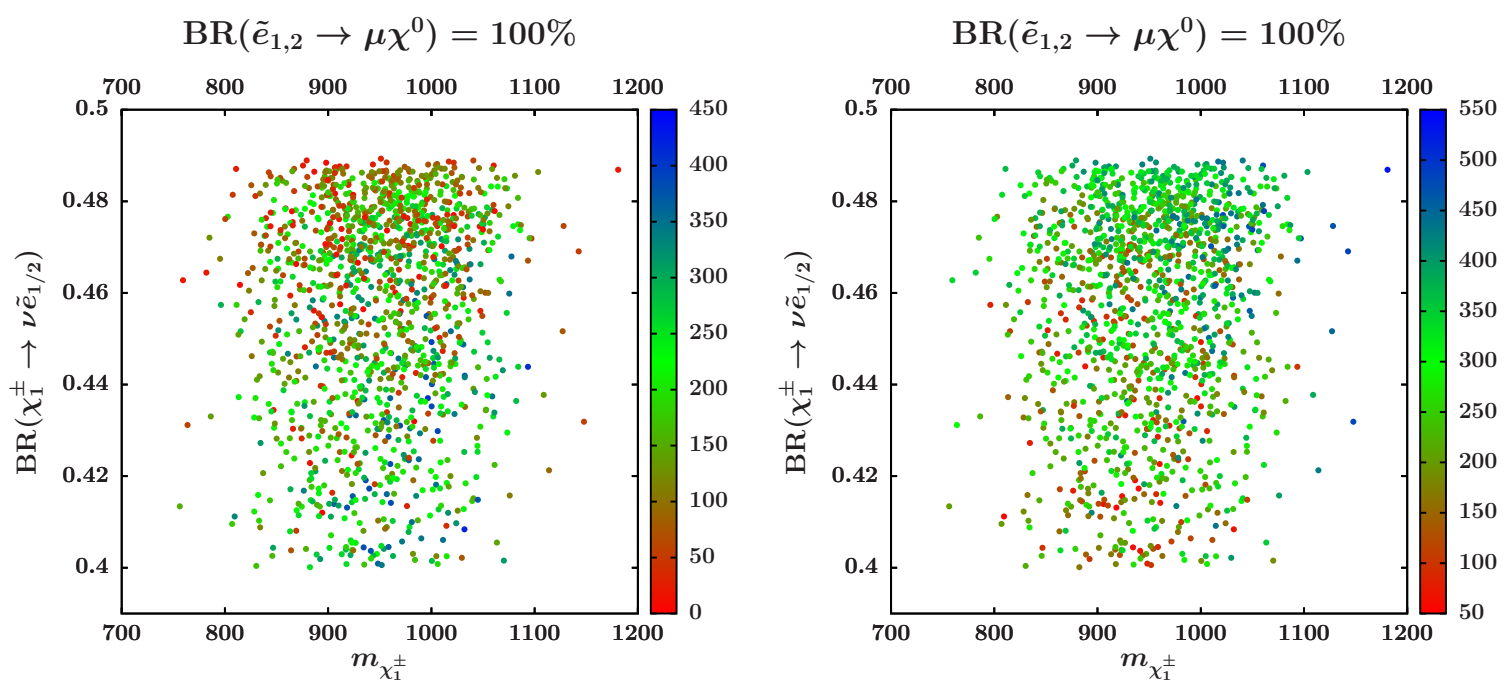

Figure 4: Same as Fig. 3 but for $\chi_{1}^{ \pm} \rightarrow \nu \tilde{e}$ with the points in the left panel color coded according to the mass difference $m_{\tilde{e}}-m_{\chi_{1}^{0}}$ while those in the right panel according to $m_{\chi_{1}^{ \pm}}-m_{\tilde{e}}$

\subsection{Decay modes for $\chi_{1}^{ \pm}$:}

The charginos decay into $l \tilde{\nu}$ and $\nu \tilde{l}$ with equal branching ratios for three generation in "flavor-democratic" simplified model. As we discussed in the previous subsection, due to 
the different smuon mixing as compared to MSSM, the charginos largely decay into $\nu \tilde{\mu}$ and $\mu \tilde{\nu}$ with a branching ratio of $50 \%$ each or the corresponding $\tau$ lepton channel.

In the right panel of Fig. 2, we show the branching ratios into different leptonic channels for $\chi_{1}^{ \pm}$as a function of $m_{\chi_{1}^{ \pm}}$. We can see that the chargino dominantly decays to muons with a very small fraction going to $\tau$ and none to electrons. Thus a three muon signal is the most likely and also the most constraining signature to look for in the trilepton searches.

In Fig. 4, we present the branching ratios of $\chi_{1}^{ \pm}$where it decays into a neutrino and a slepton $\left(\tilde{e}_{1} / \tilde{e}_{2}\right)$, where the slepton further decays into a muon and an LSP. The points are color coded according to the mass differences $m_{\tilde{e}}-m_{\chi_{1}^{0}}$ (left panel) and $m_{\chi_{1}^{ \pm}}-m_{\tilde{e}}($ right panel). These $\operatorname{BRs}\left(\chi_{1}^{ \pm} \rightarrow \nu \tilde{e}_{1 / 2} \rightarrow \nu \mu \chi_{1}^{0}\right)$ varies within $40-50 \%$ with the rest being $\chi_{1}^{ \pm} \rightarrow \mu \tilde{\nu}$, where the slepton could be degenerate with either $\chi_{1}^{0}$ or $\chi_{2}^{0}$.

\subsection{Benchmark points and models}

From the results of the previous section on the decay modes of $\chi_{1}^{ \pm}$and $\chi_{2}^{0}$, we can see that the collider constraint that is best suited to probe the chargino-neutralino pair production are the trilepton searches. In Table 1, we show three benchmark points chosen from Figs. 24. The decay properties of these points are strikingly different from the usual simplified models considered by LHC collaboration to interpret the trilepton limits. Also the mass hierarchies between sleptons and the electroweakinos are different in our model. Motivated by these benchmark points we choose the following three scenarios, or benchmark models.

- Benchmark Model 1 (BM1): This model is motivated by benchmark point 1 (BP1) where the electroweakinos dominantly decay into muons. Here sleptons are NLSP and nearly degenerate with the LSP and we assume $m_{\tilde{\nu}_{1}}=m_{\tilde{e}_{1}}=m_{\chi_{1}^{0}}$ $+10 \mathrm{GeV}$. For the branching ratios of the electroweakinos we assume $\operatorname{BR}\left(\chi_{1}^{ \pm} \rightarrow\right.$ $\left.\mu \tilde{\nu}_{1}, \nu \tilde{e}_{1}\right)=0.50$, and $\operatorname{BR}\left(\chi_{2}^{0} \rightarrow \mu \tilde{e}_{1}, \nu \tilde{\nu}\right)=0.50$; where $\operatorname{BR}\left(\tilde{e}_{1} \rightarrow \mu \chi_{1}^{0}\right)=1.0$. These assumptions apply to each point in the parameter space.

- Benchmark Model 2 (BM2): BM2 is motivated by BP2 and the decay patterns of BM2 are same as BM1 but with the choice $m_{\tilde{\nu}_{1}}=m_{\tilde{e}_{1}}=m_{\chi_{1}^{0}}+50 \mathrm{GeV}$. This choice of mass dependence can significantly change the limits on chargino masses.

- Benchmark Model 3 (BM3): BM3 is motivated by BP3 and the decay patterns of chargino and neutralino are similar to previous benchmark models. We choose the slepton mass as: $m_{\tilde{\nu}_{1}}=m_{\tilde{e}_{1}}=\left(m_{\chi_{2}^{0}}+m_{\chi_{1}^{0}}\right) / 2$. This choice of mass basically is similar to the simplified models considered by ATLAS, but BM3 is different in terms of branching ratios.

\section{Collider analysis for trilepton searches}

Both CMS and ATLAS Collaborations have searched for the EWinos with different final states $\left(2 l, 3 l\right.$, with/without taus, $l b b, l \gamma \gamma$ etc.) from direct pair production of $\chi_{1}^{ \pm} \chi_{2}^{0}$ or $\chi_{1}^{ \pm} \chi_{1}^{ \pm}[39-47]$. The results are mainly interpreted for Slepton mediated simplified model, WZ mediated simplified model and Wh mediated simplified model. In the first case, the 


\begin{tabular}{|c|c|c|c|c|}
\hline & Parameter & BP1 & BP2 & BP3 \\
\hline \multirow{11}{*}{ GUT inputs } & $m_{0}$ & 1023 & 1162 & 970 \\
\hline & $m_{1 / 2}$ & 1398 & 1544 & 1358 \\
\hline & $A_{0}$ & 36 & 1317 & 606 \\
\hline & $M_{V}$ & 329 & 324 & 746 \\
\hline & $B_{0}$ & 692 & -410 & 278 \\
\hline & $\lambda_{5}$ & -0.16 & -0.14 & -0.16 \\
\hline & $\widetilde{m}^{2}\left(\times 10^{6}\right)$ & 1.6 & 1.9 & 1.6 \\
\hline & $\widetilde{M}$ & 4.3 & 4 & -10.9 \\
\hline & $\tan \beta$ & 44.7 & 48.5 & 48.8 \\
\hline & $\lambda_{D, 2}$ & -0.39 & -0.34 & -0.38 \\
\hline & $\lambda_{L, 2}$ & -0.2 & -0.17 & -0.19 \\
\hline \multirow{7}{*}{ Pole masses } & $m_{h}$ & 124 & 123 & 123 \\
\hline & $m_{\chi_{1}^{0}}$ & 474 & 526 & 463 \\
\hline & $m_{\chi_{1}^{ \pm}}$ & 898 & 993 & 875 \\
\hline & $m_{\tilde{e}_{1}}$ & 484 & 576 & 669 \\
\hline & $m_{\tilde{e}_{2}}$ & 858 & 866 & 752 \\
\hline & $m_{\tilde{\nu}_{1}}$ & 475 & 569 & 663 \\
\hline & $m_{\tilde{t}_{R}}$ & 2021 & 2297 & 1986 \\
\hline \multirow{7}{*}{ Branching Ratios } & $\chi^{\chi_{1}^{ \pm}} \rightarrow \mu \tilde{\nu}$ & 0.5 & 0.5 & 0.5 \\
\hline & $\chi_{1}^{ \pm} \rightarrow \nu \tilde{e}_{1}$ & 0.49 & 0.48 & 0.47 \\
\hline & $\chi_{2}^{0} \rightarrow \mu \tilde{e}_{1}$ & 0.49 & 0.49 & 0.48 \\
\hline & $\chi_{2}^{0} \rightarrow \nu \tilde{\nu}$ & 0.5 & 0.5 & 0.49 \\
\hline & $\tilde{e}_{1} \rightarrow \mu \chi_{1}^{0}$ & 1.0 & 1.0 & 1.0 \\
\hline & $\delta(g-2)_{\mu}\left(\times 10^{-9}\right)$ & 2.54 & 2.23 & 2.09 \\
\hline & $\Delta m=m_{\tilde{e}_{1}}-m_{\chi_{1}^{0}}$ & 10 & 50 & $\left(m_{\chi_{2}^{0}}-m_{\chi_{1}^{0}}\right) / 2$ \\
\hline
\end{tabular}

Table 1: Benchmark points chosen such that they satisfy the constraints as described in the text as well as giving a contribution to $(g-2)_{\mu}$ that is consistent with the current $2 \sigma$ limit. All masses are in GeV.

sleptons are assumed to be lighter than $\chi_{1}^{ \pm}$and $\chi_{2}^{0}$ and this channel gives the most stringent bounds as the EWinos decay via slepton to lepton enriched final states [46]. For rest of the two cases, sleptons are assumed to be much heavier than $\chi_{1}^{ \pm}$or $\chi_{2}^{0}$ and the electroweakinos decay via real or virtual $W, Z$ and Higgs boson. In our own model, the LHC limits on gluinos from $13 \mathrm{TeV}$ data put stringent bounds on $m_{\chi_{1}^{ \pm}} \gtrsim 700 \mathrm{GeV}$ (due to High scale input) and only the trilepton analysis targeting $\chi_{1}^{ \pm} \chi_{2}^{0}$ production is sensitive to $m_{\chi_{1}^{ \pm}}>700$ $\mathrm{GeV}$ region. Hence in this analysis we only focus on the trilepton channels (dedicated signal regions for Slepton mediated simplified model). First we will briefly discuss about the $13 \mathrm{TeV}$ trilepton search analysis considered by ATLAS [46] and present our results 


\begin{tabular}{|c|c|c|c|c|c|}
\hline & SR3l-a & SR3l-b & SR3l-c & SR3l-d & SR3l-e \\
\hline$N_{\text {lepton }}$ & \multicolumn{5}{|c|}{3} \\
\hline $\mathrm{E}_{\mathrm{T}}>(\mathrm{GeV})$ & \multicolumn{5}{|c|}{130} \\
\hline$m_{T}^{\min }>(\mathrm{GeV})$ & \multicolumn{5}{|c|}{110} \\
\hline$m_{S F O S}(\mathrm{GeV})$ & \multicolumn{2}{|c|}{$<81.2$} & \multicolumn{3}{|c|}{$>101.2$} \\
\hline$p_{T}^{l_{3}}(\mathrm{GeV})$ & $20-30$ & $>30$ & $20-50$ & $50-80$ & $>80$ \\
\hline Observed events & 4 & 3 & 9 & 0 & 0 \\
\hline Total SM & $2.23 \pm 0.79$ & $2.79 \pm 0.43$ & $5.41 \pm 0.93$ & $1.42 \pm 0.38$ & $1.14 \pm 0.23$ \\
\hline
\end{tabular}

Table 2: Selection requirements for slepton mediated (3l) channel considered by ATLAS for $13 \mathrm{TeV} 36.1 \mathrm{fb}^{-1}$ data [46].

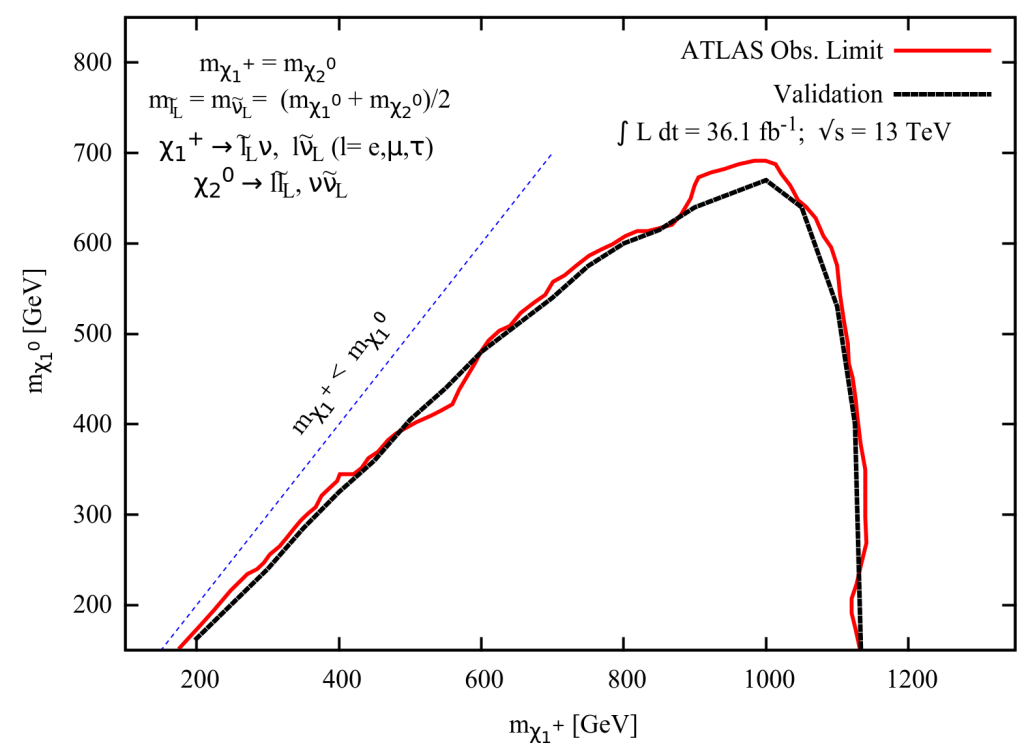

Figure 5: Validation of the ATLAS trilepton analysis for Run-II $36.1 \mathrm{fb}^{-1}$ data [46]. The exclusion limit in the $m_{\chi_{1}^{0}}-m_{\chi_{1}^{ \pm}}$plane obtained by the ATLAS Collaboration (red line) in their trilepton analysis is reproduced using similar mass relations and branching ratios of the relevant gauginos and sleptons (black line).

alongside ATLAS for validation and direct comparison.

\subsection{Validation for trilepton analysis}

In slepton $\left(\widetilde{\ell}_{L}\right)$-mediated models, it is assumed that the left handed sleptons and sneutrinos lie exactly midway between $\chi_{1}^{0}$ and $\chi_{2}^{0}, m_{\tilde{\ell}_{L}}=\left(m_{\tilde{\chi}_{1}^{\mp}}+m_{\chi_{2}^{0}}\right) / 2$, and the EWinos decay either to left handed sleptons or sneutrinos universally. Events are considered with exactly three tagged leptons (electron or muon) [46]. Event reconstruction details like electron, muon, tau and jet identification, isolation, overlap removal etc. are followed according to the ATLAS analysis as mentioned in Sec. 5 and Sec. 6 of [46]. In this trilepton analysis, a 


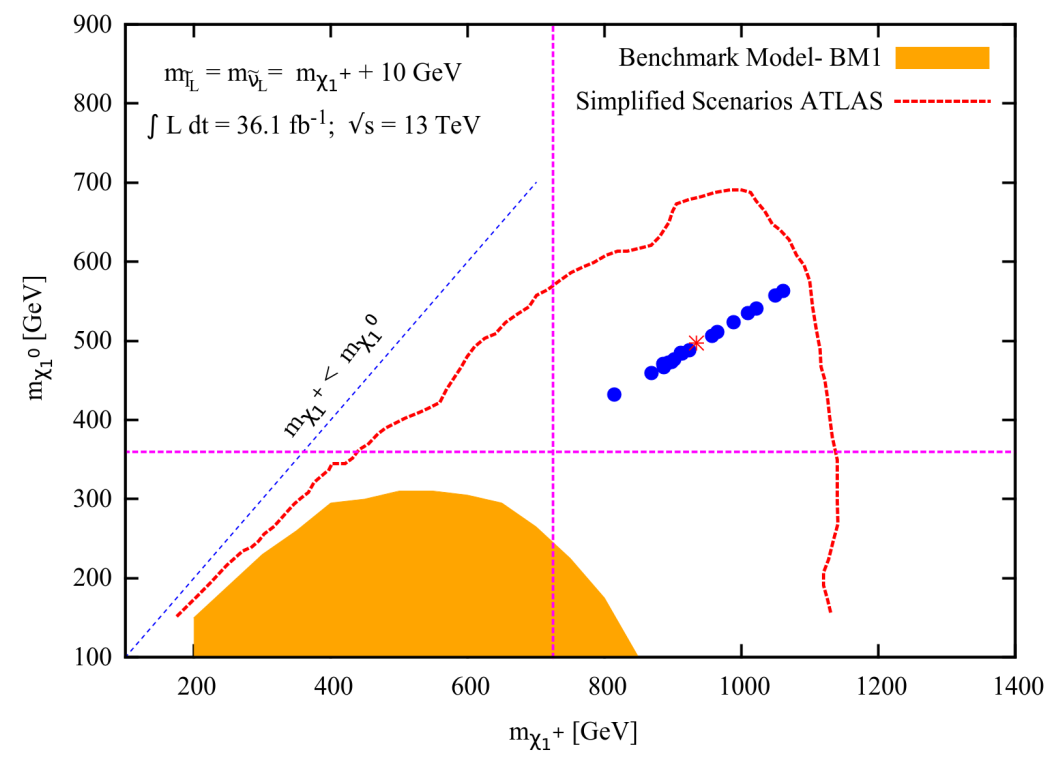

Figure 6: Limits on $m_{\chi_{1}^{ \pm}}-m_{\chi_{1}^{0}}$ plane for Benchmark Model 1 (see text). The excluded region for BM1 is shown in orange. The red dotted line represents the limit for slepton mediated simplified scenarios [46]. The magenta lines indicate the indirect lower limit on $m_{\chi_{1}^{ \pm}}$(vertical line) and $m_{\chi_{1}^{0}}$ (horizontal line) from gluino seaches in $13 \mathrm{TeV}$ data [87]. The blue points (circle) will be allowed by the New Muon $g-2$ result in a future measurement at Fermilab $[3,4]$ while the red points (star) will be ruled out, assuming that the central value of the measurement remains the same.

veto on $b$-jet is applied to all signal channels. For $b$-jets, we use the $p_{T}$ dependent $b$-tagging efficiencies obtained by ATLAS collaboration in Ref. [86].

Depending upon the requirement of $m_{S F O S}$ (invariant mass of same-flavour oppositesign (SFOS) lepton) and $p_{T}^{l_{3}}$ ( $p_{T}$ of third leading lepton), ATLAS has optimised five signal regions (SR), namely, SR3l-a to SR3l-e for Slepton mediated simplified model. The basic selection requirements for these channels, number of observed events and total SM background are listed in Table 2. In the absence of any BSM signal in all these channels, limits are set on the number of SUSY signal events at $95 \%$ confidence level (CL). For these five signal regions (SR3l-a to SR3l-e) $N_{\mathrm{BSM}}$ at $95 \%$ CL are 7.2, 5.5, 10.6, 3.0 and 3.0, respectively. The ATLAS Collaboration has translated these obtained upper limits on $N_{\mathrm{BSM}}$ into exclusion limits in the $m_{\chi_{1}^{0}}-m_{\chi_{1}^{ \pm}}$plane. In a similar way, we have also reproduced the exclusion contours obtained by ATLAS assuming similar mass relations and branching ratios of the relevant gauginos and sleptons. In order to validate our results we reproduce the exclusion contours using PYTHIA (v6.428) [88 ${ }^{3}$. We use the next-to-leading order (NLO) + next-to-leading logarithmic (NLL) chargino-neutralino pair production cross-sections given in Ref. [89], which have been calculated for $13 \mathrm{TeV}$ using the resummino code [90, 91]. For slepton mediated models, SR3l-e is the most sensitive channel for the parameter space with

\footnotetext{
${ }^{3}$ These same set-up of codes were also used in Ref. [59]
} 


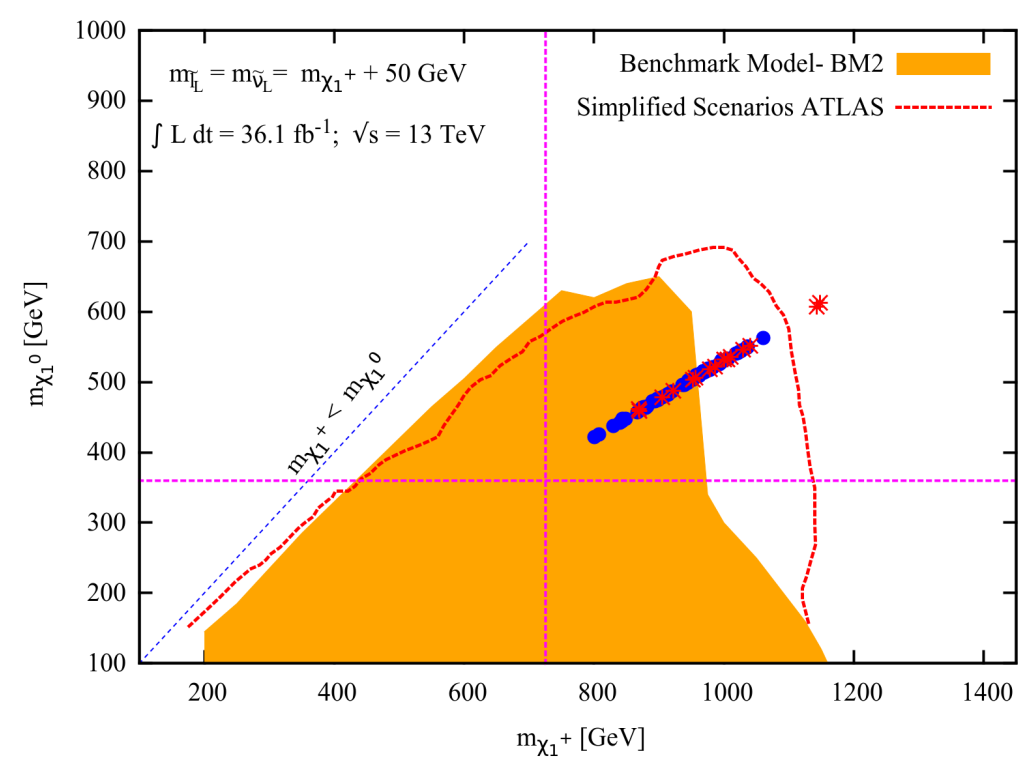

Figure 7: Same as Fig. 6 but for Benchmark Model 2 (see text).

large mass splitting between $\chi_{1}^{ \pm}$and $\chi_{1}^{0}\left(\delta m=m_{\chi_{1}^{ \pm}}-m_{\chi_{1}^{0}}\right)$. For smallest $\delta m$, low-valued $m_{S F O S} \mathrm{SR} 3 l$-a is more effective to probe the relevant parameter space.

In Fig. 5, we present the validated results for slepton mediated simplified models. The red line corresponds to $95 \%$ CL exclusion limits obtained by ATLAS and the black line corresponds to our validated results adopting the ATLAS analysis. From Fig. 5, it is evident that our validated results are in good agreement with that of ATLAS.

\subsection{New limits for benchmark models}

First we present our results for Benchmark Model 1 (BM1), where the scenarios represent slepton co-annihilation regions and due to the extreme mass degeneracy $\left(\delta m=m_{\chi_{1}^{ \pm}}-m_{\chi_{1}^{0}}\right.$ $=10 \mathrm{GeV}$ ) the leptons coming from slepton decay are very soft (below the trigger cuts). The soft leptons cause the reduction on limits on chargino masses. The orange regions in Fig. 6 are excluded from $13 \mathrm{TeV}$ data where the red dotted line represents the usual limits from simplified scenarios with the sleptons being midway between LSP and charginos. The vertical and horizontal magenta lines present the indirect limit on $m_{\chi_{1}^{ \pm}}$and $m_{\chi_{1}^{0}}$ from the gluino limits coming from $13 \mathrm{TeV}$ data [87]. The blue points (circle) are allowed by the New Muon $g-2$ result in a future measument at Fermilab [3,4] while the red points (star) are ruled out, assuming that the central value of the measurement remains the same. It is clear from Fig. 6 that for co-annihilation scenarios trilepton limits are even weaker than the indirect bounds from direct gluino searches due to the mass correlations in GUT models. It may be noted that with non-universal gaugino mass models the indirect limits from gluino searches are not valid and the models have a wide range of parameter space which are still allowed (except for the magenta regions).

The situation changes drastically if the mass difference is somewhat larger. We present the implication of trilepton data in Fig. 7 for BM2 where the mass splitting between $\chi_{1}^{ \pm}$ 


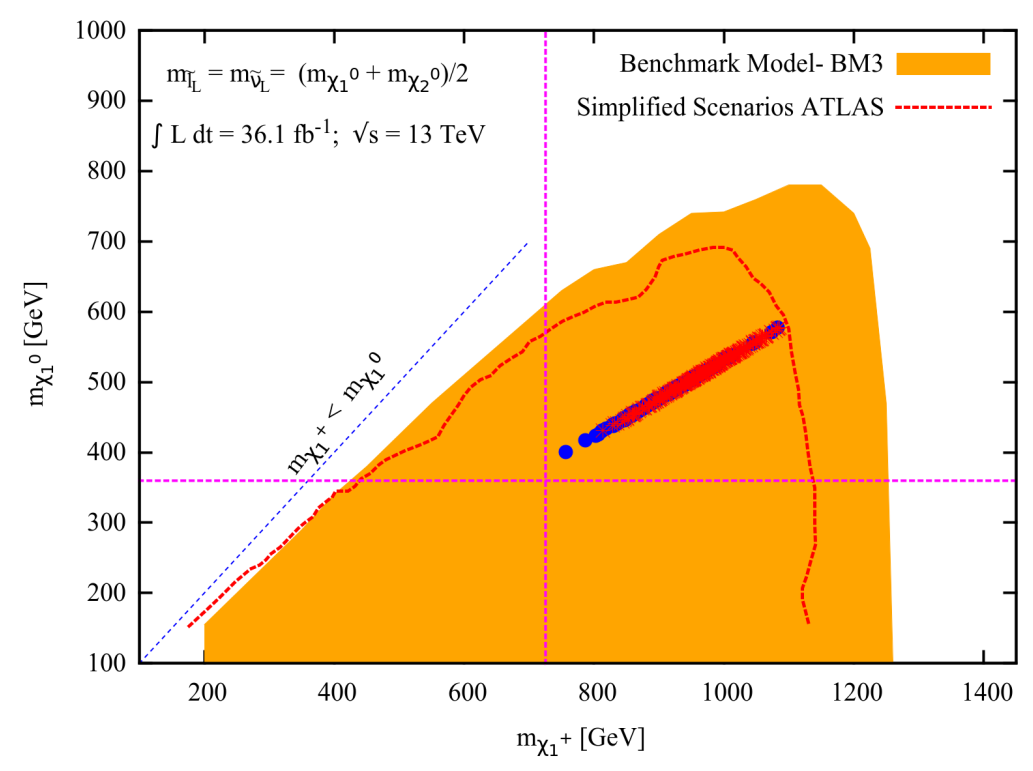

Figure 8: Same as Fig. 6 but for Benchmark Model 3 (see text).

and $\chi_{1}^{0}, \delta m=m_{\chi_{1}^{ \pm}}-m_{\chi_{1}^{0}}$, is $50 \mathrm{GeV}$. The orange regions in Fig. 7 are excluded for BM2. In some region of the parameter space the limits are stronger than usual simplified models (denoted by red line). This is simply due to the enhancement of branching ratios in our model. It may be noted that for the simplified models considered by ATLAS, the electroweakinos decay to leptonic final state universally (but for BM2, electroweakinos decay mainly to $\mu$ final states). In Fig. 7 , roughly half the points outside the orange shaded region are ruled out by Future Muon $g-2$ experiment $[3,4]$ as indicated by the red points.

In Fig. 8 we analyse the model BM3 where the sleptons are exactly midway of $\chi_{1}^{ \pm}$ and $\chi_{2}^{0}$ (same choice as simplified models). Similar to BM1 and BM2, EWinos decay also mainly to $\mu$ final states in BM3. For this model, the limits are even stronger than BM2. For light $\chi_{1}^{0}$, the limit on chargino mass extends upto $1250 \mathrm{GeV}$. Hence the current LHC data exclude all the $(g-2)_{\mu}$ allowed points which have the same characteristic like BM3.

\section{Conclusions}

In this work we have studied the VL extension of MSSM - by the addition of a pair $\mathbf{5}+\overline{\mathbf{5}}$ of $S U(5)$ which leads to an additional quark, lepton and a pair of neutrinos with corresponding squarks, sleptons and sneutrinos - in the context of $(g-2)_{\mu}$, various flavor physics constraints, DM constraints and LHC limits on squarks and gluinos. We identify that the allowed parameter space in Fig. 1 leads to chargino mass in the range of 700-1200 $\mathrm{GeV}$. The mixing of the second and third generation leptons with the extended spectrum of VL particles leads not only to an enhanced contribution to $(g-2)_{\mu}$ but also gives a very different kind of signature for electroweakino decay modes. 
To probe the allowed parameter space at the LHC, the most sensitive search will be the trilepton signal coming from chargino-neutralino pair production. For this reason we do a detailed study of relevant decay properties and the mass hierarchies in Section 4.1 and 4.2. In particular we observe that the VL extension of MSSM along with the specific choice of GUT scale parameters made here leads to a 3 muon or 3 tau final state instead of lepton universality assumed in the LHC trilepton analysis. We therefore recast the ATLAS trilepton searches in chargino-neutralino pair production using the recent Run-II data. We identify three benchmark points from the scanned dataset. To interpret the trilepton search we construct three simplified benchmark models based on these benchmark points. We observe that the slepton coannihilation scenario, i.e. BM1, is not at all sensitive to the current LHC data due to the soft nature of the lepton signal (see Fig. 6). However, the points with a relatively larger mass difference, as for example BM2, can exclude chargino mass up to $1 \mathrm{TeV}$ (see Fig. 7). The strongest constraint comes from BM3 where the slepton mass lies midway between the chargino and second lightest neutralino. For such a choice any parameter range allowed by $(g-2)_{\mu}$ data is already excluded (see Fig. 8). There still exists more than half of the parameter space that is not covered by the three benchmark models considered here, which were chosen such that they are most sensitive to the trilepton searches. For this parameter space, the allowed $2 \sigma$ range of $\delta(g-2)_{\mu}$ from the New Muon $g-2$ experiment at Fermilab [3,4] can potentially rule out more than two thirds of the region, assuming that the central value of the $(g-2)_{\mu}$ measurement remains the same. Much of this parameter space consists of tau lepton final states in chargino-neutralino pair production, which is not sensitive to the current Run-II data with $\mathcal{L}=36.1 \mathrm{fb}^{-1}[92]$. Future searches with higher luminosity for $2 \tau / 3 \tau$ signal at the LHC could potentially probe this region of the parameter space.

\section{Acknowledgments}

We would like to thank Luc Darmé and Enrico Maria Sessolo for the useful discussions in the initial stage of this work. AC and SR would like to thank Luc Darmé for helpful inputs to numerical computation. AC and LR are supported by the Lancaster-Manchester-Sheffield Consortium for Fundamental Physics under STFC Grant No. ST/L000520/1. SR and LR are supported in part by the National Science Council (NCN) research grant No. 201518-A-ST2-00748. The use of the CIS computer cluster at the National Centre for Nuclear Research in Warsaw is gratefully acknowledged.

\section{References}

[1] Muon g-2 Collaboration, G. W. Bennett et al., Final Report of the Muon E821 Anomalous Magnetic Moment Measurement at BNL, Phys. Rev. D73 (2006) 072003, [hep-ex/0602035].

[2] J. P. Miller, E. de Rafael, and B. L. Roberts, Muon (g-2): Experiment and theory, Rept. Prog. Phys. 70 (2007) 795, [hep-ph/0703049].

[3] Muon g-2 Collaboration, J. Grange et al., Muon (g-2) Technical Design Report, arXiv: 1501.06858. 
[4] Muon g-2 Collaboration, A. Chapelain, The Muon g-2 experiment at Fermilab, EPJ Web Conf. 137 (2017) 08001, [arXiv: 1701.02807].

[5] M. Endo, K. Hamaguchi, S. Iwamoto, and T. Yoshinaga, Muon g-2 vs LHC in Supersymmetric Models, JHEP 01 (2014) 123, [arXiv:1303.4256].

[6] A. Fowlie, K. Kowalska, L. Roszkowski, E. M. Sessolo, and Y.-L. S. Tsai, Dark matter and collider signatures of the MSSM, Phys. Rev. D88 (2013) 055012, [arXiv:1306.1567].

[7] M. Chakraborti, U. Chattopadhyay, A. Choudhury, A. Datta, and S. Poddar, The Electroweak Sector of the pMSSM in the Light of LHC $-8 \mathrm{TeV}$ and Other Data, JHEP 07 (2014) 019, [arXiv: 1404.4841].

[8] S. P. Das, M. Guchait, and D. P. Roy, Testing SUSY models for the muon g-2 anomaly via chargino-neutralino pair production at the LHC, Phys. Rev. D90 (2014), no. 5 055011, [arXiv: 1406.6925].

[9] M. Chakraborti, U. Chattopadhyay, A. Choudhury, A. Datta, and S. Poddar, Reduced LHC constraints for higgsino-like heavier electroweakinos, JHEP 11 (2015) 050, [arXiv: 1507.01395].

[10] M. Lindner, M. Platscher, and F. S. Queiroz, A Call for New Physics : The Muon Anomalous Magnetic Moment and Lepton Flavor Violation, arXiv:1610.06587.

[11] M. Endo, K. Hamaguchi, S. Iwamoto, and K. Yanagi, Probing minimal SUSY scenarios in the light of muon $g-2$ and dark matter, JHEP 06 (2017) 031, [arXiv:1704.05287].

[12] A. Fowlie, M. Kazana, K. Kowalska, S. Munir, L. Roszkowski, E. M. Sessolo, S. Trojanowski, and Y.-L. S. Tsai, The CMSSM Favoring New Territories: The Impact of New LHC Limits and a $125 \mathrm{GeV}$ Higgs, Phys. Rev. D86 (2012) 075010, [arXiv:1206.0264].

[13] P. Bechtle et al., Killing the cMSSM softly, Eur. Phys. J. C76 (2016), no. 2 96, [arXiv: 1508.05951].

[14] J. Cao, Z. Heng, D. Li, and J. M. Yang, Current experimental constraints on the lightest Higgs boson mass in the constrained MSSM, Phys. Lett. B710 (2012) 665-670, [arXiv: 1112.4391].

[15] S. Mohanty, S. Rao, and D. P. Roy, Reconciling the muon $g-2$ and dark matter relic density with the LHC results in nonuniversal gaugino mass models, JHEP 09 (2013) 027, [arXiv: 1303.5830].

[16] S. Akula and P. Nath, Gluino-driven radiative breaking, Higgs boson mass, muon g-2, and the Higgs diphoton decay in supergravity unification, Phys. Rev. D87 (2013), no. 11 115022, [arXiv:1304.5526].

[17] J. Chakrabortty, S. Mohanty, and S. Rao, Non-universal gaugino mass GUT models in the light of dark matter and LHC constraints, JHEP 02 (2014) 074, [arXiv:1310.3620].

[18] K. Kowalska, L. Roszkowski, E. M. Sessolo, and A. J. Williams, GUT-inspired SUSY and the muon g-2 anomaly: prospects for LHC 14 TeV, JHEP 06 (2015) 020, [arXiv:1503.08219].

[19] J. Chakrabortty, A. Choudhury, and S. Mondal, Non-universal Gaugino mass models under the lamppost of muon (g-2), JHEP 07 (2015) 038, [arXiv: 1503.08703].

[20] A. S. Belyaev, J. E. Camargo-Molina, S. F. King, D. J. Miller, A. P. Morais, and P. B. Schaefers, A to $Z$ of the Muon Anomalous Magnetic Moment in the MSSM with Pati-Salam at the GUT scale, JHEP 06 (2016) 142, [arXiv:1605.02072]. 
[21] N. Okada and H. M. Tran, 125 GeV Higgs boson mass and muon $g-2$ in 5D MSSM, Phys. Rev. D94 (2016), no. 7 075016, [arXiv: 1606.05329].

[22] T. Fukuyama, N. Okada, and H. M. Tran, Sparticle spectroscopy of the minimal SO(10) model, Phys. Lett. B767 (2017) 295-302, [arXiv:1611.08341].

[23] M. Endo, K. Hamaguchi, S. Iwamoto, and N. Yokozaki, Higgs mass, muon g-2, and LHC prospects in gauge mediation models with vector-like matters, Phys. Rev. D85 (2012) 095012, [arXiv: 1112.5653].

[24] M. Endo, K. Hamaguchi, S. Iwamoto, and N. Yokozaki, Higgs Mass and Muon Anomalous Magnetic Moment in Supersymmetric Models with Vector-Like Matters, Phys. Rev. D84 (2011) 075017, [arXiv:1108.3071].

[25] R. Dermisek and A. Raval, Explanation of the Muon g-2 Anomaly with Vectorlike Leptons and its Implications for Higgs Decays, Phys. Rev. D88 (2013) 013017, [arXiv:1305.3522].

[26] R. Dermisek, A. Raval, and S. Shin, Effects of vectorlike leptons on $h \rightarrow 4 \ell$ and the connection to the muon g-2 anomaly, Phys. Rev. D90 (2014), no. 3 034023, [arXiv: 1406.7018].

[27] I. Gogoladze, Q. Shafi, and C. S. Un, Reconciling the muon g-2, a 125 GeV Higgs boson, and dark matter in gauge mediation models, Phys. Rev. D92 (2015), no. 11 115014, [arXiv: 1509.07906].

[28] A. Aboubrahim, T. Ibrahim, and P. Nath, Leptonic $g-2$ moments, CP phases and the Higgs boson mass constraint, Phys. Rev. D94 (2016), no. 1 015032, [arXiv:1606.08336].

[29] M. Nishida and K. Yoshioka, Higgs Boson Mass and Muon g-2 with Strongly Coupled Vector-like Generations, Phys. Rev. D94 (2016), no. 9 095022, [arXiv:1605.06675].

[30] T. Higaki, M. Nishida, and N. Takeda, Flavor Structure, Higgs boson mass and Dark Matter in Supersymmetric Model with Vector-like Generations, arXiv:1611.04322.

[31] E. Megias, M. Quiros, and L. Salas, $g_{\mu}-2$ from Vector-Like Leptons in Warped Space, JHEP 05 (2017) 016, [arXiv: 1701.05072].

[32] A. Choudhury, L. Darmé, L. Roszkowski, E. M. Sessolo, and S. Trojanowski, Muon g-2 and related phenomenology in constrained vector-like extensions of the MSSM, JHEP 05 (2017) 072, [arXiv: 1701.08778].

[33] P. W. Graham, A. Ismail, S. Rajendran, and P. Saraswat, A Little Solution to the Little Hierarchy Problem: A Vector-like Generation, Phys. Rev. D81 (2010) 055016, [arXiv: 0910.3020].

[34] S. P. Martin, Extra vector-like matter and the lightest Higgs scalar boson mass in low-energy supersymmetry, Phys. Rev. D81 (2010) 035004, [arXiv:0910.2732].

[35] C. Faroughy and K. Grizzard, Raising the Higgs mass in supersymmetry with $t$ - $t^{\prime}$ mixing, Phys. Rev. D90 (2014), no. 3 035024, [arXiv: 1405.4116].

[36] Z. Lalak, M. Lewicki, and J. D. Wells, Higgs boson mass and high-luminosity LHC probes of supersymmetry with vectorlike top quark, Phys. Rev. D91 (2015), no. 9 095022, [arXiv: 1502.05702].

[37] K. Nickel and F. Staub, Precise determination of the Higgs mass in supersymmetric models with vectorlike tops and the impact on naturalness in minimal GMSB, JHEP 07 (2015) 139, [arXiv: 1505.06077]. 
[38] R. Barbieri, D. Buttazzo, L. J. Hall, and D. Marzocca, Higgs mass and unified gauge coupling in the NMSSM with Vector Matter, JHEP 07 (2016) 067, [arXiv:1603.00718].

[39] ATLAS Collaboration, G. Aad et al., Search for direct production of charginos and neutralinos in events with three leptons and missing transverse momentum in $\sqrt{s}=8 T e V p p$ collisions with the ATLAS detector, JHEP 04 (2014) 169, [arXiv: 1402.7029].

[40] ATLAS Collaboration, G. Aad et al., Search for direct production of charginos, neutralinos and sleptons in final states with two leptons and missing transverse momentum in $p p$ collisions at $\sqrt{s}=8 \mathrm{TeV}$ with the ATLAS detector, JHEP 05 (2014) 071, [arXiv: 1403.5294].

[41] ATLAS Collaboration, G. Aad et al., Search for the direct production of charginos, neutralinos and staus in final states with at least two hadronically decaying taus and missing transverse momentum in pp collisions at $\sqrt{s}=8 \mathrm{TeV}$ with the ATLAS detector, JHEP 10 (2014) 096, [arXiv: 1407.0350].

[42] ATLAS Collaboration, G. Aad et al., Search for direct pair production of a chargino and a neutralino decaying to the $125 \mathrm{GeV}$ Higgs boson in $\sqrt{s}=8 \mathrm{TeV}$ pp collisions with the ATLAS detector, Eur. Phys. J. C75 (2015), no. 5 208, [arXiv:1501.07110].

[43] ATLAS Collaboration, G. Aad et al., Search for the electroweak production of supersymmetric particles in $\sqrt{s}=8$ TeV pp collisions with the ATLAS detector, Phys. Rev. D93 (2016), no. 5 052002, [arXiv: 1509.07152].

[44] CMS Collaboration, V. Khachatryan et al., Searches for electroweak neutralino and chargino production in channels with Higgs, $Z$, and $W$ bosons in pp collisions at 8 TeV, Phys. Rev. D90 (2014), no. 9 092007, [arXiv:1409.3168].

[45] CMS Collaboration, V. Khachatryan et al., Searches for electroweak production of charginos, neutralinos, and sleptons decaying to leptons and W, Z, and Higgs bosons in pp collisions at 8 TeV, Eur. Phys. J. C74 (2014), no. 9 3036, [arXiv:1405.7570].

[46] ATLAS Collaboration Collaboration, Search for electroweak production of supersymmetric particles in the two and three lepton final state at $\sqrt{s}=13 \mathrm{TeV}$ with the ATLAS detector, Tech. Rep. ATLAS-CONF-2017-039, CERN, Geneva, Jun, 2017.

[47] CMS Collaboration Collaboration, Combined search for electroweak production of charginos and neutralinos in pp collisions at $\sqrt{s}=13 \mathrm{TeV}$, Tech. Rep. CMS-PAS-SUS-17-004, CERN, Geneva, 2017.

[48] A. Bharucha, S. Heinemeyer, and F. von der Pahlen, Direct Chargino-Neutralino Production at the LHC: Interpreting the Exclusion Limits in the Complex MSSM, Eur. Phys. J. C73 (2013), no. 11 2629, [arXiv:1307.4237].

[49] K. Howe and P. Saraswat, Excess Higgs Production in Neutralino Decays, JHEP 10 (2012) 065, [arXiv: 1208.1542].

[50] P. Schwaller and J. Zurita, Compressed electroweakino spectra at the LHC, JHEP 03 (2014) 060, [arXiv: 1312.7350].

[51] D. Ghosh, M. Guchait, and D. Sengupta, Higgs Signal in Chargino-Neutralino Production at the LHC, Eur. Phys. J. C72 (2012) 2141, [arXiv:1202.4937].

[52] J. Eckel, M. J. Ramsey-Musolf, W. Shepherd, and S. Su, Impact of LSP Character on Slepton Reach at the LHC, JHEP 11 (2014) 117, [arXiv:1408.2841]. 
[53] A. Choudhury and A. Datta, Neutralino dark matter confronted by the LHC constraints on Electroweak SUSY signals, JHEP 09 (2013) 119, [arXiv:1305.0928].

[54] C. Han, L. Wu, J. M. Yang, M. Zhang, and Y. Zhang, New approach for detecting a compressed bino/wino at the LHC, Phys. Rev. D91 (2015) 055030, [arXiv:1409.4533].

[55] F. Yu, Anatomizing Exotic Production of the Higgs Boson, Phys. Rev. D90 (2014), no. 1 015009, [arXiv: 1404.2924].

[56] A. Papaefstathiou, K. Sakurai, and M. Takeuchi, Higgs boson to di-tau channel in Chargino-Neutralino searches at the LHC, JHEP 08 (2014) 176, [arXiv: 1404.1077].

[57] M. A. Ajaib, B. Dutta, T. Ghosh, I. Gogoladze, and Q. Shafi, Neutralinos and sleptons at the LHC in light of muon $(g-2)_{\mu}$, Phys. Rev. D92 (2015), no. 7 075033, [arXiv:1505.05896].

[58] T. Han, S. Padhi, and S. Su, Electroweakinos in the Light of the Higgs Boson, Phys. Rev. D88 (2013), no. 11 115010, [arXiv:1309.5966].

[59] A. Choudhury and S. Mondal, Revisiting the Exclusion Limits from Direct Chargino-Neutralino Production at the LHC, Phys. Rev. D94 (2016), no. 5 055024, [arXiv: 1603.05502].

[60] Q.-F. Xiang, X.-J. Bi, P.-F. Yin, and Z.-H. Yu, Searching for Singlino-Higgsino Dark Matter in the NMSSM, Phys. Rev. D94 (2016), no. 5 055031, [arXiv:1606.02149].

[61] A. Datta, N. Ganguly, and S. Poddar, New Limits on Heavier Electroweakinos and their LHC Signatures, Phys. Lett. B763 (2016) 213-217, [arXiv:1606.04391].

[62] K. Hagiwara, K. Ma, and S. Mukhopadhyay, Closing in on the chargino contribution to the muon g-2 in the MSSM: current LHC constraints, arXiv:1706.09313.

[63] A. Arbey, M. Boudaud, F. Mahmoudi, and G. Robbins, Robustness of dark matter constraints and interplay with collider searches for New Physics, arXiv:1707.00426.

[64] M. Chakraborti, A. Datta, N. Ganguly, and S. Poddar, Multilepton signals of heavier electroweakinos at the LHC, arXiv:1707.04410.

[65] J. Cao, Y. He, L. Shang, W. Su, and Y. Zhang, Testing the light dark matter scenario of the MSSM at the LHC, JHEP 03 (2016) 207, [arXiv:1511.05386].

[66] A. Kobakhidze, M. Talia, and L. Wu, Probing the MSSM explanation of the muon g-2 anomaly in dark matter experiments and at a 100 TeV pp collider, Phys. Rev. D95 (2017), no. 5 055023, [arXiv:1608.03641].

[67] F. Feroz, M. P. Hobson, and M. Bridges, MultiNest: an efficient and robust Bayesian inference tool for cosmology and particle physics, Mon. Not. Roy. Astron. Soc. 398 (2009) 1601-1614, [arXiv: 0809.3437].

[68] F. Staub, SARAH 4: A tool for (not only SUSY) model builders, Comput.Phys.Commun. 185 (2014) 1773-1790.

[69] F. Staub, Automatic Calculation of supersymmetric Renormalization Group Equations and Self Energies, Comput.Phys.Commun. 182 (2011) 808-833.

[70] W. Porod and F. Staub, SPheno 3.1: Extensions including flavour, CP-phases and models beyond the MSSM, Comput. Phys. Commun. 183 (2012) 2458-2469, [arXiv:1104.1573].

[71] F. Staub, T. Ohl, W. Porod, and C. Speckner, A Tool Box for Implementing Supersymmetric Models, Comput. Phys. Commun. 183 (2012) 2165-2206, [arXiv:1109.5147]. 
[72] W. Porod, F. Staub, and A. Vicente, A Flavor Kit for BSM models, Eur. Phys. J. C74 (2014), no. 8 2992, [arXiv:1405.1434].

[73] G. Belanger, F. Boudjema, A. Pukhov, and A. Semenov, micrOMEGAs 3 : A program for calculating dark matter observables, Comput. Phys. Commun. 185 (2014) 960-985, [arXiv: 1305.0237].

[74] P. Bechtle, S. Heinemeyer, O. Stl, T. Stefaniak, and G. Weiglein, HiggsSignals: Confronting arbitrary Higgs sectors with measurements at the Tevatron and the LHC, Eur. Phys. J. C74 (2014), no. 2 2711, [arXiv:1305.1933].

[75] P. Bechtle, O. Brein, S. Heinemeyer, G. Weiglein, and K. E. Williams, HiggsBounds: Confronting Arbitrary Higgs Sectors with Exclusion Bounds from LEP and the Tevatron, Comput. Phys. Commun. 181 (2010) 138-167, [arXiv:0811.4169].

[76] P. Bechtle, O. Brein, S. Heinemeyer, G. Weiglein, and K. E. Williams, HiggsBounds 2.0.0: Confronting Neutral and Charged Higgs Sector Predictions with Exclusion Bounds from LEP and the Tevatron, Comput. Phys. Commun. 182 (2011) 2605-2631, [arXiv:1102.1898].

[77] P. Bechtle, O. Brein, S. Heinemeyer, O. Stl, T. Stefaniak, G. Weiglein, and K. E. Williams, HiggsBounds - 4: Improved Tests of Extended Higgs Sectors against Exclusion Bounds from LEP, the Tevatron and the LHC, Eur. Phys. J. C74 (2014), no. 3 2693, [arXiv:1311.0055].

[78] Y. Amhis et al., Averages of b-hadron, c-hadron, and $\tau$-lepton properties as of summer 2016, arXiv: 1612.07233.

[79] Belle Collaboration, I. Adachi et al., Evidence for $B^{-} \rightarrow \tau^{-} \bar{\nu}_{\tau}$ with a Hadronic Tagging Method Using the Full Data Sample of Belle, Phys. Rev. Lett. 110 (2013), no. 13 131801, [arXiv: 1208.4678].

[80] Particle Data Group Collaboration, C. Patrignani et al., Review of Particle Physics, Chin. Phys. C40 (2016), no. 10100001.

[81] LHCb Collaboration, R. Aaij et al., Measurement of the $B_{s}^{0} \rightarrow \mu^{+} \mu^{-}$branching fraction and search for $B^{0} \rightarrow \mu^{+} \mu^{-}$decays at the LHCb experiment, Phys. Rev. Lett. 111 (2013) 101805, [arXiv: 1307.5024].

[82] CMS Collaboration, S. Chatrchyan et al., Measurement of the B(s) to mu+ mu- branching fraction and search for B0 to mu+ mu- with the CMS Experiment, Phys. Rev. Lett. 111 (2013) 101804, [arXiv:1307.5025].

[83] BaBar Collaboration, B. Aubert et al., Searches for Lepton Flavor Violation in the Decays $\tau^{ \pm} \rightarrow e^{ \pm} \gamma$ and $\tau^{ \pm} \rightarrow \mu^{ \pm} \gamma$, Phys. Rev. Lett. 104 (2010) 021802, [arXiv:0908. 2381].

[84] Planck Collaboration, P. A. R. Ade et al., Planck 2015 results. XIII. Cosmological parameters, Astron. Astrophys. 594 (2016) A13, [arXiv:1502.01589].

[85] LUX Collaboration, D. S. Akerib et al., Results from a search for dark matter in the complete LUX exposure, Phys. Rev. Lett. 118 (2017), no. 2 021303, [arXiv:1608. 07648].

[86] ATLAS Collaboration Collaboration, Measurement of the b-tag Efficiency in a Sample of Jets Containing Muons with $5 \mathrm{fb}^{-1}$ of Data from the ATLAS Detector, Tech. Rep. ATLAS-CONF-2012-043, CERN, Geneva, Mar, 2012.

[87] ATLAS Collaboration Collaboration, Search for squarks and gluinos in final states with jets and missing transverse momentum using $36 \mathrm{fb}^{-1}$ of $\sqrt{s}=13$ TeV pp collision data with the ATLAS detector, Tech. Rep. ATLAS-CONF-2017-022, CERN, Geneva, Apr, 2017. 
[88] T. Sjostrand, S. Mrenna, and P. Z. Skands, PYTHIA 6.4 Physics and Manual, JHEP 05 (2006) 026, [hep-ph/0603175].

[89] https://twiki.cern.ch/twiki/bin/view/LHCPhysics/SUSYCrossSections.

[90] B. Fuks, M. Klasen, D. R. Lamprea, and M. Rothering, Gaugino production in proton-proton collisions at a center-of-mass energy of 8 TeV, JHEP 10 (2012) 081, [arXiv:1207.2159].

[91] B. Fuks, M. Klasen, D. R. Lamprea, and M. Rothering, Precision predictions for electroweak superpartner production at hadron colliders with Resummino, Eur. Phys. J. C73 (2013) 2480, [arXiv: 1304.0790].

[92] ATLAS Collaboration Collaboration, Search for the direct production of charginos and neutralinos in final states with tau leptons in $\sqrt{s}=13 \mathrm{TeV} p p$ collisions with the ATLAS detector, Tech. Rep. ATLAS-CONF-2017-035, CERN, Geneva, May, 2017. 